\title{
Dynamic-arc respiratory-gated stereotactic radiotherapy - technique presentation
}

\author{
Grzegorz Woźniak , Łukasz Dolla², Krzysztof Ślosarek ${ }^{2}$, ${ }^{3}$ Barbara Bekman², \\ Tomasz Latusek ${ }^{1}$, Grzegorz Głowacki ${ }^{1}$
}

Main advancements in radiation treatment in recent years have included the introduction of dynamic techniques and $4 \mathrm{D}$ radiotherapy. The treatment of movable tumors relies on two important techniques: gating and tracking. The limitation of the former is the relatively short duration of the respiratory phase during which the radiation can be delivered and the need to teach the patient to breathe in accordance with the correct pattern. At the same time, certain clinical situations require the use of dynamic techniques. Intensity modulated radiotherapy (IMRT), combined with gantry rotation, forms the basis for the VMAT technique. The procedure usually takes a shorter time to complete than other dynamic techniques, which considerably improves patient comfort. The recently introduced True Beam ${ }^{\mathrm{TM}}$ accelerator employs all the latest innovations in terms of dose-rate modeling and respiratory gating.

NOWOTWORY J Oncol 2017; 67, 5: 297-300

Key words: respiratory gating, stereotactic radiotherapy, dynamic arc, VMAT

\section{Description of the method}

Respiratory-gated stereotactic radiotherapy with the use of the dynamic arcs (RA - Rapid $\operatorname{Arc}^{\circledR}$ ) technique, implemented in daily practice at the Gliwice center, requires additional patient preparation procedures. These involve patient immobilization, respiratory phase imaging, the delineation of target volume and critical structures with respect to the selected breathing phase, radiotherapy planning, and relevant dosimetric measurements.

Patients are immobilized on the table with the aid of vacuum mattresses, which are easy to employ, can be reused multiple times, and do not restrict respiratory movements, in contrast to devices such as thermoplastic masks, for instance. In theory, radiotherapy can be performed without patient immobilization, but mattresses help reduce torso bending and rotation and thus considerably improve patient comfort during a procedure that can often take up to an hour to complete.
The most important preliminary stage involves the detection and imaging of the appropriate respiratory phase with a Siemens Somatom CT scanner. Scans are usually taken at intervals of 1-3 $\mathrm{mm}$, both before and after the radiocontrast is administered, and $4 \mathrm{D}$ radiotherapy is made possible by a detection system that relies on an infrared camera to register respiratory movements by tracking the displacements of a metaplex slab phantom with six fluorescent markers inserted near the epigastric region. The RPM (Real-time Positioning Management) system analyzes and records the patient's breathing pattern as a sinusoid that indicates the point of maximum inhalation and exhalation. Based on this curve, an appropriate respiratory phase is then selected for therapy. The final exhalation phase is usually considered the longest and the most stable; accordingly, this is when treatment is administered. To guarantee the accuracy of the $C T$ image reconstruction for each phase, which affects the quality of the final scan, it is essential to make sure

\footnotetext{
${ }^{1}$ Radiotherapy Department, Maria Skłodowska-Curie Cancer Center and Institute of Oncology, Gliwice Branch, Poland ${ }^{2}$ Radiotherapy and Brachytherapy Planning Department, Maria Skłodowska-Curie Cancer Center and Institute of Oncology, Gliwice Branch, Poland

${ }^{3}$ Humanitas University in Sosnowiec, Poland
} 
that the patient's breathing is stable and regular. Since the RPM system allows for manual adjustment, slight breathing anomalies can be disregarded. If greater disruptions occur, however, the test should be stopped and repeated.

The CT lab of the Treatment Planning Unit uses a retrospective respiratory-gated CT testing method, which means that the entire respiratory phase is scanned; the RPM system first records a breathing curve for the patient, which then serves as the basis for selecting the appropriate phase for image processing and reconstruction. If doubts arise with respect to the exact size of the target volume, an additional MRI scan is performed. MRI tests are very advantageous, especially in liver tumors, but also show important limitations in terms of subsequent fusion with CT scans. As they require a special coil, they cannot be performed with the aid of immobilization mattresses. In addition, imaging sequences in MRI are longer than in CT, which leads to motion-related image distortions. Despite the gating options currently in use, organ locations in MRI scans often fail to match completely with those in CT images, which causes discrepancies during subsequent fusion.

Based on CT scans and their fusion with MRI images, target volumes and critical organs are then delineated, and the treatment plan is elaborated.

Alongside standard radiation energies, the Varian TrueBeam $^{\mathrm{TM}}$ linear accelerator also includes two flattening filter-free beams of 6 and $10 \mathrm{MV}$, which allow to generate dose rates of $1400 \mathrm{MU} / \mathrm{min}$ and $2400 \mathrm{MU} / \mathrm{min}$, respectively. In addition, the high speed of the multileaf collimator (MLC) and the option of planning based on the arc technique with dose rate modulation have made it possible to use the VMAT (volumetric modulated arc therapy) in 4D radiotherapy. Several arcs are usually used, mainly due to the limited number of monitor units that can be measured during a single gantry rotation cycle. In addition, multiple arcs that differ in collimator angles often yield more accurate isodose curves, because of the greater variety of MLC leaf positions.

For every plan prepared with the volumetric modulated arc technique (VMAT), the dose distribution should be verified through dosimetry before the treatment is initiated. This is done with the aid of Sun Nuclear's ArcCheck ${ }^{\circledR}$ cylindrical multidetector array, which allows to carry out accurate measurements and reconstruct the 3D dose distribution pattern in the phantom. Due to its submilimeter accuracy, additional equipment geometry tests are also required before every radiotherapy session. Apart from standard accelerator efficiency tests, a modified Winston-Lutz test is carried out to control the position of the isocenter of the gantry and the correlated isocenter of the $\mathrm{OBI}$ image verification system with maximum precision.

Once the patient has been immobilized on the table, verification scans are taken. As a standard practice, two kilovoltage orthogonal X-ray projections are used. The True-
Beam $^{\mathrm{TM}}$ accelerator allows to coordinate imaging time with the previously selected respiratory phase (and the DRR image generated by the treatment planning system). This allows to avoid respiratory motion-related discrepancies in reference point location. Another verification method relies on CBCT (cone beam computer tomography), which involves taking CT scans in the area of interest with a cone beam of orthovoltage $\mathrm{X}$-rays. The OBI system generator fixed to the arm of the treatment device completes a single rotation around the patient; the kilovoltage beam is recorded as it penetrates the tissues, processed, and converted into a CT image. A lower exposure than that of classical computer tomography is enough to yield a image. The $3 \mathrm{D}$ reconstruction of CT scans significantly improves the accuracy of stereotactic radiotherapy, especially when the treatment table permits rotary movement [1]. However, the longer total scanning time considerably limits the application of CBCT in respiratory-gated radiotherapy, since the images cannot be taken in the same respiratory phase. For this reason, the system is not commonly used in this form of treatment.

Treatment delivery is automatic. During rotation by a fixed angle, radiation exposure is only switched on in the pre-defined breathing phase, i.e. when the tumor is located within the field of the radiation beam. Radiation is suspended once breathing moves on to the next phase and the gantry is moved back, so that it can deliver radiation again during the next respiratory cycle and move by a further angle down the pre-defined arc. The movement of the slab phantom placed in the epigastric region is tracked in three dimensions by infrared cameras throughout the procedure. If breathing anomalies exceed accepted tolerance limits, treatment is stopped until correct breathing is restored. Importantly, flattening filter-free (FFF) VMAT treatment involves a high-intensity dose that changes with the changing rotational speed of the accelerator's arm. This significantly shortens the procedure time in comparison with conformal radiotherapy techniques.

\section{Discussion}

Stereotactic radiotherapy (SR) is currently recognized as one of the most effective radiation treatment methods [2,3]. Initially used in the treatment of benign intracranial tumors (Gamma Knife) [4], it is now emoloyed in a variety of intracranial and extracranial cancers. Indications for stereotactic therapy include primary, relapsed (also benign), and secondary brain tumors that do not qualify for surgery [5-10]. Extracranially, SR is used to treat small, sharply delineated tumors in various parts of the body, including primary tumors and metastatic lymph nodes in the head and neck area, tumors of the chest, abdomen, and pelvis (especially the prostate gland), as well as lesions found in limbs and bones [3,11-14]. In the case of respiratory moving tumors, the technique can be successfully applied 
in the treatment of lung and liver cancers, often constituting an alternative to radical surgery or palliative care $[2,15-18]$. Tumors located in organs affected by respiratory motion on both sides of the diaphragm (mediastinum, kidneys, adrenal glands, spleen) can also be treated with radiosurgery with the use of respiratory gating or tracking [19-22]. The basic limitation of the method is the size and location of the tumor. Delivering a single high dose of radiation to a small tumor volume often has the biological effect comparable to its surgical ablation (hence the term radiosurgery or radioablation) [23-25]. High doses, however, are equally dangerous to healthy tissues, which means they can only be used for small tumors (usually up to $4 \mathrm{~cm}$ ). A greater target volume means that more healthy tissues will absorb the beam and treatment toxicity will increase. Tumor location and the possibility of delivering high radiation doses near critical structures constitute another significant limitation of stereotactic therapy. The risk of geographical error can be reduced through the key procedures of patient immobilization and position verification [26]. In addition, dynamic techniques (IMRS, VMAT) allow to accurately modulate dose distributions, achieve high dose gradients, and escalate the dose in tumors near vital organs [27, 28]. The use of image-guided verification (IGRT - image guided radiotherapy) often provides a very accurate estimate of tumor location with respect to anatomical structures that should be protected with particular attention.

Technologies generating energetically homogeneous beams have been perfected over many years, based on the premise that tumor cells are evenly distributed and show similar radiation sensitivity [29-31]. In order to cause uniform and even damage to these cells, the argument went, an even and uniform beam of radiation should be delivered. Successive years, however, delivered new data, indicating that clonogenic tumor cells distribution is not homogenous and show varying degrees of radiosensitivity determined by different biological phenomena, such as hypoxia [31]. Accordingly, constructors of radiotherapy equipment no longer tried to generate a uniform radiation beam; to the contrary, new technologies relied on pre-existing heterogeneous dose distributions or even deliberately created them by altering (modulating) beam intensity.

In any accelerator, electrons stopped on the anode shield generate a heterogeneous radiation beam: the dose is highest near the axis and decreases as the beam radiates away. The beam profile is often uniformized by a cone filter, which results in a flattened, uniform dose distribution throughout the area delimited by the collimator jaws [32]. Unfortunately, the procedure always involves the weakening of radiation intensity and a decrease in dose rates. In contrast, using multileaf collimators to modulate the beam profile makes it possible to achieve a non-uniform dose distribution in target volumes of any shape. One of the chief drawbacks of this technique (IMRT) has to do with long exposure times due to the small size of radiation fields (a larger field is divided into smaller beams that change over time) [33]. Changes in the approach to dose heterogeneity also altered the way in which new accelerators are constructed. Instead of using a cone filter to model a uniform radiation beam in the collimator, the cone was removed and the beam is modified as it leaves the shield. Removing the filter, which normally absorbs a large portion of initial radiation (from 50 to $90 \%$ ), allows to achieve a high dose intensity, which in turn shortens exposure times (FFF beams).

Two techniques are used in the radiotherapy of motion-dependent areas: gating and tracking [34, 35]. The main limitation of the former is the relatively short duration (gate) of the respiratory phase during which the dose can be delivered. Low dose rates and the insufficient speed of MLC leaves in earlier accelerators limited the application of dynamic techniques in respiratory gating. Thanks to the high doses generated in filter-free accelerator heads, a high amount of energy can now be delivered in a short time (as short as the respiratory gate), which makes it potentially available for use in gating.

The TrueBeam ${ }^{\mathrm{TM}}$ accelerator allows to use FFF beams (FFF - flattening filter free) with dose rates of up to 2400 $\mathrm{MU} / \mathrm{min}$ (in a standard cone-filter accelerator, the corresponding value equals $600 \mathrm{MU} / \mathrm{min}$ ). In addition, the use of the VMAT (or RapidArc ${ }^{\circledR}$ technology in the Varian company nomenclature), which combines gantry rotation over partial or complete arcs with the modulation of dose rates and collimator leaf movement, allows to deliver gated radiotherapy through a dynamic technique.

The RPM RapidArc ${ }^{\circledR}$ method shares all the advantages and drawbacks of other dynamic techniques. In some cases, conformal plans yield similar target volume and critical structure parameters, while requiring less time to complete. The VMAT technique, however, makes it possible to treat several neighboring tumors based on the same reference point, that is difficult in conformal techniques and often proves impossible, especially if the goal is to deliver a different dose to each tumor. The VMAT technique shows a significant advantage in the treatment of tumors situated near critical structures, but higher efficiency in this case is usually achieved at the expense of lower dose uniformity and a greater volume of healthy tissues that absorb radiation. The VMAT technique is particularly beneficial when a HD collimator (with leaf width of $2.5 \mathrm{~mm}$ ) can be used to fine-tune the shape of the beam to match the spatial form of the tumor.

The essential advantage of the VMAT technique has to do with procedure duration. Using FFF beams with high dose rates considerably reduces effective radiation time. Thanks to varying dose intensity (delivery time decreases two- to fourfold), it can be reduced by as much as $30 \%$. 
The difference is keenly felt by the patient, especially when several tumors are treated at the same time. Shorter therapy sessions should be viewed from the perspective of treatment precision.

In the case of the FFF-VMAT technique, verification of patient's set up takes longer because the system can take scans only in certain phases of the respiratory cycle, which means that, in a sense, it needs to "wait" for the patient to start breathing along a specific pre-defined pattern. In addition, the time is extended if respiratory anomalies occur; in this case, treatment is suspended until normal breathing is restored.

\section{Conclusion}

To sum up, respiratory-gated radiotherapy is a commonly recognized treatment method for lung and liver cancers, which may also be successfully used in other tumors located near the diaphragm. The proximity of critical organs in the treatment area often makes VMAT radiotherapy a valuable treatment option and even a necessity if patient comfort and safety are to be ensured.

\section{Conflict of interest: none declared}

\section{Grzegorz Woźniak, MD, PhD}

Maria Skłodowska-Curie Cancer Center and Institute of Oncology Gliwice Branch

Radiotherapy Department

ul. Wybrzeże Armii Krajowej 15

44-100 Gliwice, Poland

e-mail: grzegorz.wozniak@io.gliwice.pl

Received: 16 Oct 2016

Accepted: 8 Jun 2017

\section{References}

1. Purdie TG, Bissonnette JP, Franks K et al. Cone-beam computed tomography for on-line image guidance of lung stereotactic radiotherapy: localization, verification, and intrafraction tumor position. Int J Radiat Oncol Biol Phys 2007; 68: 243-252.

2. Carey Sampson M, Katz A, Constine LS. Stereotactic body radiation therapy for extracranial oligometastases: does the sword have a double edge? Semin Radiat Oncol 2006; 16: 67-76.

3. Ricardi U, Badellino S, Filippi AR. Clinical applications of stereotactic radiation therapy for oligometastatic cancer patients: a disease-oriented approach. J Radiat Res 2016; 57 (Suppl 1): i58-i68.

4. Leksell L. Sterotaxic radiosurgery in trigeminal neuralgia. Acta Chir Scand 1971; 137: 311-314.

5. Kondziolka $D$, Shin SM, Brunswick $A$ et al. The biology of radiosurgery and its clinical applications for brain tumors. Neuro Oncol 2015; 17:29-44.

6. Badiyan SN, Regine WF, Mehta M. Stereotactic radiosurgery for treatment of brain metastases. J Oncol Pract 2016; 12: 703-712.

7. Soliman H, Das S, Larson DA et al. Stereotactic radiosurgery (SRS) in the modern management of patients with brain metastases. Oncotarget 2016; 7: 12318-12330.

8. Minniti G, Clarke E, Scaringi C et al. Stereotactic radiotherapy and radiosurgery for non-functioning and secreting pituitary adenomas. Rep Pract Oncol Radiother 2016; 21: 370-378.

9. Redmond KJ, Mehta M. Stereotactic radiosurgery for glioblastoma. Cureus 2015; 7: e413.

10. Mansouri A, Guha D, Klironomos $G$ et al. Stereotactic radiosurgery for intracranial meningiomas: current concepts and future perspectives. Neurosurgery 2015; 76: 362-371.
11. Clemente S, Nigro R, Oliviero C et al. Role of technical aspects of hypofractionated radiation therapy treatment of prostate cancer: a review. Int J Radiat Oncol Biol Phys 2015; 91: 182-195.

12. Tan TJ, Siva S, Foroudi F et al. Stereotactic body radiotherapy for primary prostate cancer: a systematic review. J Med Imaging Radiat Oncol 2014; 58: 601-611.

13. Tseng CL, Eppinga W, Charest-Morin R et al. Spine stereotactic body radiotherapy: indications, outcomes, and points of caution. Global Spine J 2017; 7: 179-197.

14. Redmond KJ, Sahgal A, Foote $M$ et al. Single versus multiple session stereotactic body radiotherapy for spinal metastasis: the risk-benefit ratio. Future Oncol 2015; 11: 2405-2415.

15. Miszczyk L, Majewski W, Matuszewski M et al. Stereotaktyczna pozaczaszkowa radiochirurgia guzów wątroby z bramkowaniem oddechowym wiązki promieniowania prezentacja metody. Przegl Lek 2007; 64: 454-461.

16. Timmerman $\mathrm{R}$, Paulus $\mathrm{R}$, Galvin J et al. Stereotactic body radiation therapy for inoperable early stage lung cancer. JAMA 2010; 303: 1070-1076.

17. Fakiris AJ, McGarry RC, Yiannoutsos CT et al. Stereotactic body radiation therapy for early-stage non-small-cell lung carcinoma: four-year results of a prospective phase II study. Int J Radiat Oncol Biol Phys 2009; 75: 677-682.

18. Kavanagh BD, Schefter TE, Cardenes HR et al. Interim analysis of a prospective phase I/II trial of SBRT for liver metastases. Acta Oncol 2006; 45: 848-855.

19. Pham D, Kron T, Bressel M et al. Image guidance and stabilization for stereotactic ablative body radiation therapy (SABR) treatment of primary kidney cancer. Pract Radiat Oncol 2015; 5: e597-e605.

20. Barney BM, Olivier KR, Macdonald OK et al. Clinical outcomes and dosimetric considerations using stereotactic body radiotherapy for abdominopelvic tumors. Am J Clin Oncol 2012; 35: 537-542.

21. Haidenberger $\mathrm{A}$, Heidorn $\mathrm{SC}$, Kremer $\mathrm{N}$ et al. Robotic radiosurgery for adrenal gland metastases. Cureus 2017; 9: e1120.

22. Scorsetti M, Alongi F, Filippi AR et al. Long-term local control achieved after hypofractionated stereotactic body radiotherapy for adrenal gland metastases: a retrospective analysis of 34 patients. Acta Oncol 2012; 51:618-623.

23. Timmerman RD. An overview of hypofractionation and introduction to this issue of seminars in radiation oncology. Semin Radiat Oncol 2008; 18: 215-222.

24. Niranjan A, Gobbel GT, Kondziolka D et al. Experimental radiobiological investigations into radiosurgery: present understanding and future directions. Neurosurgery 2004; 55: 495-504.

25. De La Fuente Herman T, Vlachaki MT, Herman TS et al. Stereotactic body radiation therapy (SBRT) and respiratory gating in lung cancer: dosimetric and radiobiological considerations. J Appl Clin Med Phys 2010; 11: 3133.

26. Goitein M. Organ and tumor motion: an overview. Semin Radiat Oncol 2004; 14: 2-9.

27. Ong CL, Verbakel WF, Cuijpers JP et al. Stereotactic radiotherapy for peripheral lung tumors: a comparison of volumetric modulated arc therapy with 3 other delivery techniques. Radiother Oncol 2010; 97: 437-442.

28. Merrow CE, Wang IZ, Podgorsak MB. A dosimetric evaluation of VMAT for the treatment of non-small cell lung cancer. J Appl Clin Med Phys 2012; 14: 4110.

29. Joiner MC. Quantifying cell kill and cell survival. In: Basic clinical radiobiology. 4th ed. Joiner M, van der Kogel A (eds.). London: Edward Arnold, 2009: 41-55.

30. Zips D. Tumour growth and response to radiation. In: Basic clinical radiobiology. 4th ed. Joiner M, van der Kogel A (eds.). London: Edward Arnold, 2009: 78-92.

31. Maciejewski B, Withers R. From conventional averages to individual dose painting in radiotherapy for human tumours: challenge to non-uniformity. Nowotwory J Oncol 2004; 54: 1-10.

32. $\quad$ Łobodziec W. Dozymetria promieniowania jonizujacego $w$ radioterapii. 2nd ed. Katowice:Wydawnictwo Uniwersytetu Śląskiego, 1999: 20-27.

33. Ślosarek K. Podstawy planowania leczenia w radioterapii. Gliwice: PTO, 2007.

34. Mageras GS, Yorke, E. Deep inspiration breath hold and respiratory gating strategies for reducing organ motion in radiation treatment. Semin Radiat Oncol 2004; 14: 65-75.

35. Dawson LA, Balter JM. Interventions to reduce organ motion effects in radiation delivery. Semin Radiat Oncol 2004; 14: 76-80. 